\title{
Vapnik-Chervonenkis Density on Indiscernible Sequences, Stability, and the Maximum Property
}

\author{
Hunter Johnson
}

\begin{abstract}
This paper presents some finite combinatorics of set systems with applications to model theory, particularly the study of dependent theories. There are two main results. First, we give a way of producing lower bounds on $\mathrm{VC}_{\text {ind }}$-density and use it to compute the exact $\mathrm{VC}_{\text {ind }}$-density of polynomial inequalities and a variety of geometric set families. The main technical tool used is the notion of a maximum set system, which we juxtapose to indiscernibles. In the second part of the paper we give a maximum set system analogue to Shelah's characterization of stability using indiscernible sequences.
\end{abstract}

\section{Introduction}

In the recent past, there have been a number of papers relating various measures of the combinatorial structure of NIP theories to one another (see Aschenbrenner, Dolich, Haskell, MacPherson, and Starchenko [1], Guingona and Hill [5], and Kaplan, Onshuus, and Usvyatsov [7]). One fact which emerged from this is the close relation of dp-rank and VC-density restricted to indiscernible sequences. Guingona and Hill have used the term $\mathrm{VC}_{\text {ind }}$-density to describe $\mathrm{VC}$-density restricted to indiscernibles. At the end of their paper [5], Guingona and Hill ask if there is a useful characterization of when a formula has $\mathrm{VC}_{\text {ind }}$-density equal to $\mathrm{VC}$-density. We offer such a characterization below (see Corollary 3.8) and use it to compute the exact $\mathrm{VC}_{\text {ind }}$-density of certain formulas.

A separate goal of this paper is to show how maximum set systems can in many cases be used as more accessible surrogates for indiscernible sequences. To this end we, translate Shelah's well-known characterization of stability in terms of indiscernible sets to a version involving maximum set systems.

Received January 4, 2013; accepted August 8, 2013

2010 Mathematics Subject Classification: Primary 03C45; Secondary 68R05

Keywords: VC-density, VC-dimension, NIP, stability

(C) 2015 by University of Notre Dame 10.1215/00294527-3153597 


\section{Notation}

Let there be a fixed complete theory $T$, with a large saturated model $\mathfrak{M}=\langle\mathrm{M}, \ldots\rangle$. All sets and models, unless otherwise stated, are assumed to be elementarily embedded in the model $\mathfrak{M}$. We write formulas in partitioned form $\varphi(\mathbf{x}, \mathbf{y})$, where $\mathbf{x}=\left\langle x_{1}, \ldots, x_{l}\right\rangle$, and $\mathbf{y}=\left\langle y_{1}, \ldots, y_{k}\right\rangle$. We use $\mathcal{P}(X)$ to denote the power set of $X$.

For $A \subseteq \mathbf{M}^{|\mathbf{y}|}$ and $\mathbf{b} \in \mathbf{M}^{|\mathbf{x}|}$,

$$
\varphi(\mathbf{b}, A):=\{\mathbf{a} \in A: \models \varphi(\mathbf{b}, \mathbf{a})\} .
$$

We use $S_{\varphi}(A)$ for $A \subseteq \mathrm{M}^{|\mathbf{y}|}$ to denote the set of $\varphi$-types over $A$, where a $\varphi$-type over $A$ is a maximal consistent set of the form $\left\{ \pm \varphi(\mathbf{x}, \mathbf{a}): \mathbf{a} \in \mathrm{M}^{|\mathbf{y}|}\right\}$. We let $\left.S_{\varphi}(A)\right|_{B}=\left\{t p_{\varphi}(\mathbf{b} / A): \mathbf{b} \in B\right\}$ when $B \subseteq \mathbf{M}^{|\mathbf{x}|}$. For an individual type $p \in S_{\varphi}(A)$, we often identify $p$ and the set of its positive parameter instances $\{\mathbf{a} \in A: \varphi(\mathbf{x}, \mathbf{a}) \in p\}$ without further comment. Similarly, we sometimes identify $\left.S_{\varphi}(A)\right|_{B}$ and $\{\varphi(\mathbf{b}, A): \mathbf{b} \in B\}$, as in the following definition.

Definition 2.1 Let $\mathcal{C} \subseteq \mathcal{P}\left(\mathrm{M}^{|\mathbf{x}|}\right)$. We will say that $\varphi(\mathbf{x}, \mathbf{y})$ traces $\ell$ if for some $A \subseteq \mathrm{M}^{|\mathbf{x}|}, \mathcal{C} \subseteq S_{\varphi}(A)$.

We now give some purely combinatorial definitions. For the rest of this section

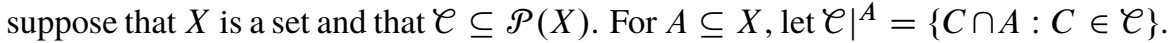
Say that $\mathcal{C}$ shatters $A$ if $\left.\mathcal{C}\right|^{A}=\mathcal{P}(A)$.

Definition 2.2 The Vapnik-Chervonenkis $(V C)$ dimension of $\mathcal{C}$, denoted $\operatorname{VC}(\mathcal{C})$, is $|A|$, where $A \subseteq X$ is of maximum finite cardinality such that $\mathcal{C}$ shatters $A$.

If the VC-dimension of $\mathcal{C}$ does not exist, we write $\mathrm{VC}(\mathcal{C})=\infty$. The VC-dimension was first considered in Vapnik and Chervonenkis [16] and was introduced into model theory by Laskowski [11]. The following notion of a maximum VC family was defined by Welzl [17].

Definition 2.3 Say that $\ell$ is $d$-maximum for $d \in \omega$ if, for any finite $A \subseteq X$, $|\mathcal{C}|^{A} \mid=\left(\begin{array}{c}|A| \\ \leq d\end{array}\right)$.

Here $\left(\begin{array}{c}n \\ \leq k\end{array}\right)$ is shorthand for $\sum_{i=0}^{k}\left(\begin{array}{c}n \\ i\end{array}\right)$ if $k<n$ and $2^{n}$ otherwise.

Lemma 2.4 (Sauer's lemma [13], Shelah [14]) If $\mathcal{C}$ has $\operatorname{VC}(\mathcal{C})=d$, then for any finite $A \subseteq X$,

$$
|\mathcal{E}|^{A} \mid \leq\left(\begin{array}{c}
|A| \\
\leq d
\end{array}\right) .
$$

Thus a $d$-maximum set system is "extremal" among set systems of VC-dimension $d$. These set systems are highly structured and well understood (see Floyd [4] and Kuzmin and Warmuth [10]). There are several examples that arise from natural algebraic situations. In fact it is conjectured that all $d$-maximum set systems arise from (or embed naturally in) arrangements of half-spaces, either in a Euclidean or hyperbolic space (see Rubinstein and Rubinstein [12]).

It is easy to see that if $\mathcal{C}$ is $d$-maximum on $X$, and $A \subseteq X$ has $|A|=d+1$, then

$$
\left.C\right|^{A}=\mathcal{P}(A) \backslash\{C\}
$$

for some $C \subseteq A$. Floyd [4] calls such a $C$ the forbidden label of $\mathcal{C}$ on $A$. 
Let $[X]^{m}:=\{A \subseteq X:|A|=m\}$. For a fixed $d$-maximum $\mathcal{C} \subseteq \mathcal{P}(X)$, associate with each $A \in[X]^{d+1}$ the forbidden label $C_{A} \subseteq A$, where $\left.\mathcal{C}\right|^{A}=\mathscr{P}(A) \backslash\left\{C_{A}\right\}$.

Floyd proves the following.

Proposition 2.5 ([4, Lemma 3.15]) On a finite domain $X$, any $d$-maximum set system $C$ is characterized by its forbidden labels, in the sense that $\forall B \subseteq X$,

$$
B \in \mathcal{C} \Longleftrightarrow \forall A \in[X]^{d+1}(B \cap A) \neq C_{A} .
$$

Proof Left-to-right is obvious. For right-to-left, let $B$ satisfy the given conditions.

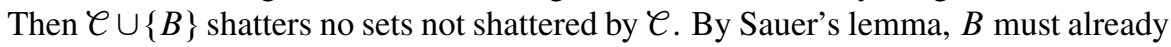
be in $\varphi$.

We now define the notion of a forbidden code, which is essentially the "form" of a forbidden label when an ordering is present.

Let $\mathfrak{L}_{\mathcal{C}}(X)=\left\{C_{A}: A \in[X]^{d+1}\right\}$ denote the forbidden labels of $\mathcal{C}$ on $X$. Let $<$ be a fixed but arbitrary linear order on $X$. For each $C_{A} \in \mathfrak{L}_{\mathcal{C}}(X)$, let $\overline{C_{A}}=\left\langle t_{0}, \ldots, t_{d}\right\rangle$, where each $t_{i} \in\{0,1\}$, and $t_{i}=1$ if and only if the $i$ th element of $A$ is in $C_{A}$. Define $\overline{\mathfrak{L}_{e}(X)}=\left\{\overline{C_{A}}: C_{A} \in \mathfrak{L}_{e}(X)\right\}$.

We will refer to $\overline{\mathfrak{L}_{e}(X)}$ as the set of forbidden codes on $X$ for $\ell$, with respect to $<$. When a maximum set system has a unique forbidden code, that code determines everything about the system at the level of finite traces. Technically, we say that the system is finitely characterized by the code.

Definition 2.6 The set system $\mathcal{C} \mathscr{P}(X)$ is finitely characterized by $\eta \in 2^{d+1}$ if for any finite $X_{0} \subseteq X$, and $A \subseteq X_{0}$, the following are equivalent:

1. $A \in \boldsymbol{C}^{X_{0}}$,

2. there are not elements $a_{0}<\cdots<a_{d}$ in $X_{0}$ such that $a_{i} \in A \Longleftrightarrow \eta(i)=1$.

There is a natural way in which forbidden codes can serve as combinatorial invariants for finite unions of points and <-convex subsets in $X$. To see this, suppose that $(X,<)$ is a dense and complete linear order and that $B \subseteq X$ is a finite union of convex subsets. Let $d$ be the number of boundary points of $B$. We can imagine that $B$ is defined by some $L_{<}=\{<\}$formula $\psi\left(x, c_{1}, \ldots, c_{d}\right)$ with $c_{1}<\cdots<c_{d} \in X$. Define $\mathcal{F}(B)=\left\{\psi\left(X, c_{1}^{\prime}, \ldots, c_{d}^{\prime}\right): c_{1}^{\prime}<\cdots<c_{d}^{\prime} \in X\right\}$. Intuitively, the elements of $\mathcal{F}(B) \subseteq \mathscr{P}(X)$ are the "homeomorphic images" of $B$ in $(X,<)$. In Johnson [8] we show that $\mathcal{F}(B)$ is finitely characterized by some $\eta \in 2^{d+1}$.

Define the genus of $B$, denoted $\mathbb{G}(B)$, to be the $\eta \in 2^{d+1}$ that finitely characterizes $\mathscr{F}(B)$. Equivalently, define $\mathbb{G}(B)$ to be any $\eta \in 2^{d+1}$ such that there are no $a_{0}<\cdots<a_{d}$ in $X$ such that $a_{i} \in B$ if and only if $\eta(i)=1$, for all $i=0, \ldots, d$.

In [8] we show that such an $\eta$ exists and is unique, as well as the further fact that for any $\eta \in 2^{<\omega}$, there is some $B \subseteq X$ such that $\mathbb{G}(B)=\eta$. Simple rules for computing genus are given in Table 1 .

To give an example of applying the table, suppose that $X=\mathbb{R}$ and that $<$ is the usual ordering. Then the genus of the point $\{0\}$ is $\langle 11\rangle$, and the genus of the interval $(0,1)$ is $\langle 101\rangle$. Conversely, to consider all subsets of $\mathbb{R}$ with genus $\langle 11\rangle$, let $\ell$ be all the singletons. Similarly, the collection of all subsets of genus $\langle 101\rangle$ is exactly the set of all infinite convex subsets which are not coinitial or cofinal.

The assumption that $(X,<)$ is complete was made to give a clear presentation of the genus concept and is sufficient for this paper. One can, however, define the genus 
Table 1 A key for assigning forbidden codes to unions of convex sets.

\begin{tabular}{|l|l|}
\hline Code & Translation \\
\hline$\langle 1 \ldots\rangle$ & do nothing \\
$\langle 0 \ldots\rangle$ & $(-\infty, \ldots$ \\
$\langle\ldots 0, \ldots\rangle$ & remove point \\
$\langle\ldots 0,1 \ldots\rangle$ & end interval \\
$\langle\ldots 1,0 \ldots\rangle$ & begin interval \\
$\langle\ldots 1,1 \ldots\rangle$ & add point \\
$\langle\ldots 0\rangle$ & $\ldots, \infty)$ \\
$\langle\ldots 1\rangle$ & do nothing \\
\hline
\end{tabular}

of $B \subseteq X$ on other orders by considering the shortest $\eta \in 2^{<\omega}$ which $B$ does not induce, sidestepping the issue of boundary points.

The link between genus and forbidden codes is given in the following theorem.

Theorem 2.7 Suppose that $(X,<)$ is a complete dense linear order without endpoints. If $\eta \in 2^{d+1}$ and $\complement=\{C \subseteq X: \mathbb{G}(C)=\eta\}$, then $\zeta$ is $d$-maximum on $X$ and $\overline{\mathfrak{L e}_{e}(X)}=\{\eta\}$.

Proof We provide a sketch of the proof that $\ell$ is $d$-maximum, which is very similar to the well-known proof that unions of intervals are maximum.

Let $X_{0} \subseteq X$ be finite, and let $\ell=\{C \subseteq X: \mathbb{G}(C)=\eta\}$. Let $a:=\max X_{0}$, and let $X_{0}^{a}=X_{0} \backslash\{a\}$. Define $\mathcal{C}^{a}=\left\{\left.C \in \mathcal{C}\right|^{X_{0}^{a}}:\left.\left.C \cup\{a\} \in \mathcal{C}\right|^{X_{0}} \& C \in \mathcal{C}\right|^{X_{0}}\right\}$. By induction on $\left|X_{0}\right|$ and $d, e^{a}$ is $(d-1)$-maximum and $\ell^{X_{0}^{a}}$ is $d$-maximum. Then

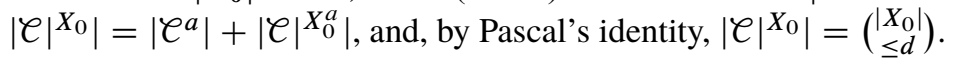

\section{Results}

3.1 VCm and $\mathbf{V C}_{\text {ind }}$-density We now apply the above to achieve our results. Recall the following definitions.

Definition 3.1 A formula $\varphi(\mathbf{x}, \mathbf{y})$ has $V C$-density at most $r$ for $r \in \mathbb{R}$ if there is $K \in \omega$ such that for every finite $A \subseteq \mathrm{M}^{|\mathbf{y}|},\left|S_{\varphi}(A)\right|<K \cdot|A|^{r}$. We denote this by $\operatorname{VCd}(\varphi) \leq r$.

Definition 3.2 A formula $\varphi(\mathbf{x}, \mathbf{y})$ has $\mathrm{VC}_{\text {ind }}$-density at most $r$ for $r \in \mathbb{R}$ if there is $K \in \omega$ such that for every finite and indiscernible sequence $\overline{\mathbf{b}}=\left\langle\mathbf{b}_{i}: i<N\right\rangle \in$ $\mathbf{M}^{|\mathbf{y}| \cdot N},\left|S_{\varphi}(\operatorname{range}(\overline{\mathbf{b}}))\right|<K \cdot N^{r}$. We denote this by $\operatorname{VCd}_{\text {ind }}(\varphi) \leq r$.

The study of VC-density has emerged several times in model theory. See [1] for historical remarks.

There has been some study of the fact that frequently $\operatorname{VCd}(\varphi)$ is bounded by a simple (and uniform) function of $|\mathbf{x}|$ (see [1] and Johnson and Laskowski [9]). When this is true, it justifies the heuristic practice of "parameter counting" to guess the complexity of set systems. Guingona and Hill showed that in a $d p$-minimal theory, $\operatorname{VCd}_{\text {ind }}(\varphi) \leq|\mathbf{x}|$. Thus there is interest in bounding $\operatorname{VCd}(\varphi)$ by a function of $\operatorname{VCd}_{\text {ind }}(\varphi)$ (obviously $\operatorname{VCd}(\varphi) \geq \operatorname{VCd}_{\text {ind }}(\varphi)$ ). This may not be possible in general, but we now show a practicable route to achieving it for a given formula.

Definition 3.3 For a set $A \subseteq \mathrm{M}^{|\mathbf{y}|}$, we denote the traces of $\varphi(\mathbf{x}, \mathbf{y})$ on $A$ by

$$
\operatorname{Tr}(\varphi, A)=\mathcal{P}\left(S_{\varphi}(A)\right) .
$$


We refer to the traces of $\varphi$ on sets of cardinality $\kappa$ by

$$
\operatorname{Tr}_{\kappa}(\varphi)=\bigcup_{A \in\left[\mathrm{M}^{|y|} \mid\right]^{\kappa}} \operatorname{Tr}(\varphi, A) .
$$

The following is easy, but interesting, because the collection of $d$-maximum set systems would seem, a priori, to be very diverse. It also informs Definition 3.5.

Lemma 3.4 Let $\kappa$ be infinite. For each $d \in \omega$, there is some $\mathcal{C} \in \operatorname{Tr}_{\kappa}(\varphi)$ such that $\mathcal{C}$ is $d$-maximum if and only if for each $n \in \omega, n \geq d$, there is $\ell_{n} \in \operatorname{Tr}_{n}(\varphi)$ such that $\mathcal{C}_{n}$ is d-maximum.

Proof For the right-to-left direction, it is easily seen that the property of being $d$-maximum is elementary. Apply the compactness theorem and the saturation of $\mathfrak{M}$. For left-to-right, note that if $\mathcal{C}$ is $d$-maximum and $X^{\prime} \subseteq X$ with $\left|X^{\prime}\right|=n, n \geq d$, then $\left.e\right|^{X^{\prime}}$ is $d$-maximum.

As a consequence of Lemma 3.4, if $\kappa$ is infinite, then there exists $\ell \in \operatorname{Tr}_{\kappa}(\varphi)$ which is $d$-maximum if and only if there is $\ell^{\prime} \in \operatorname{Tr}_{\aleph_{0}}(\varphi)$ such that $\ell^{\prime}$ is $d$-maximum.

Definition 3.5 The VC-maximum dimension of $\varphi$ is defined by

$$
\operatorname{VCm}(\varphi):=\max \left\{d \in \omega: \exists C \in \operatorname{Tr}_{\aleph_{0}}(\varphi) \text { s.t. } \mathcal{C} \text { is } d \text {-maximum }\right\} .
$$

Note that the definition of VC-maximum dimension does not use model theory.

If $\left\langle\mathbf{a}_{i}\right\rangle_{i \in I}$ is a sequence of indiscernibles and $(I,<)$ is a complete and dense linear order without endpoints, for $B \subseteq\left\langle\mathbf{a}_{i}\right\rangle_{i \in I}$, define $\mathbb{G}(B)$ to be the genus of $\left\{i \in I: \mathbf{a}_{i} \in B\right\} \subseteq I$. With these assumptions, for $m \in \omega$, make the definition

$$
P_{m}(B)=\left\{\rho \in 2^{m}: \exists i_{0}<\cdots<i_{m-1} \in I: \mathbf{a}_{i_{j}} \in B \Longleftrightarrow \rho(j)=1\right\} .
$$

If $\mu \in 2^{k}, \eta \in 2^{l}$, and $l \leq k$, write $\eta \unlhd \mu$ if $\eta$ is a subsequence of $\mu$, meaning that for some order-preserving embedding $f: l \rightarrow k$ (where $k$ and $l$ are regarded as ordinals) $\forall i \in l, \mu(f(i))=\eta(i)$.

We observe that

$$
\forall \rho \in 2^{m}, \quad \rho \in P_{m}(B) \Longleftrightarrow \mathbb{G}(B) \not \rho .
$$

Assume in Lemma 3.6 that the formula $\varphi(\mathbf{x}, \mathbf{y})$ is NIP.

Lemma 3.6 (Transfer lemma) Let $\left\langle\mathbf{a}_{i}\right\rangle_{i \in I}$ be a sequence of indiscernibles, where $(I,<)$ is a complete and dense linear order without endpoints. Assume that $B \subseteq\left\langle\mathbf{a}_{i}\right\rangle_{i \in I}$ is defined by $\varphi\left(\left\langle\mathbf{a}_{i}\right\rangle_{i \in I}, \mathbf{c}\right), A \subseteq\left\langle\mathbf{a}_{i}\right\rangle_{i \in I}$, and $A^{\prime} \subseteq A$ can be traced as $A^{\prime}=A \cap B^{\prime}$, where $B^{\prime} \subseteq\left\langle\mathbf{a}_{i}\right\rangle_{i \in I}$ is such that $\mathbb{G}\left(B^{\prime}\right)=\mathbb{G}(B)$. Then, there exists $\mathbf{c}^{\prime} \in \mathrm{M}^{|\mathbf{y}|}$ such that $\varphi\left(A, \mathbf{c}^{\prime}\right)=A^{\prime}$.

Proof First, consider the case in which $A$ is finite.

Let $m=|A|$, and suppose that $\mathbf{a}_{i_{0}}<\cdots<\mathbf{a}_{i_{m-1}}$ is an enumeration of $A$. Since $\mathbb{G}\left(B^{\prime}\right)=\mathbb{G}(B)$, we have $P_{m}(B)=P_{m}\left(B^{\prime}\right)$ by (1). Let $\rho \in P_{m}\left(B^{\prime}\right)$ be such that for each $j=0, \ldots, m-1, \mathbf{a}_{i_{j}} \in A^{\prime} \Longleftrightarrow \rho(j)=1$. Then $\rho \in P_{m}(B)$, and for some $\mathbf{a}_{k_{0}}<\cdots<\mathbf{a}_{k_{m-1}}$ in $\left\langle\mathbf{a}_{i}\right\rangle_{i \in I}$,

$$
\mathfrak{M} \models \bigwedge_{j=0}^{m-1} \varphi\left(\mathbf{a}_{k_{j}}, \mathbf{c}\right)^{\rho(j)}
$$


and thus

$$
\mathfrak{M} \models \exists \mathbf{y} \bigwedge_{j=0}^{m-1} \varphi\left(\mathbf{a}_{k_{j}}, \mathbf{y}\right)^{\rho(j)} .
$$

Then, by indiscernibility,

$$
\mathfrak{M} \models \exists \mathbf{y} \bigwedge_{j=0}^{m-1} \varphi\left(\mathbf{a}_{i_{j}}, \mathbf{y}\right)^{\rho(j)},
$$

and the witnessing $\mathbf{c}^{\prime}$ is the desired parameter.

The case in which $A$ is infinite now follows by compactness and the saturation of $\mathfrak{M}$.

Theorem 3.7 For any $\varphi(\mathbf{x}, \mathbf{y}), \operatorname{VCm}(\varphi)=\operatorname{VCd}_{\text {ind }}(\varphi)$.

Proof First, note that we always have $\operatorname{VCm}(\varphi) \geq 0$ and $\operatorname{VCd}_{\text {ind }}(\varphi) \geq 0$.

Now suppose that $\operatorname{VCd}_{\text {ind }}(\varphi) \geq d$, for some positive $d \in \omega$. Let $0<\epsilon<1 / 2$. By compactness, Ramsey's theorem, and saturation of the monster model, there is some indiscernible sequence $\left\langle\mathbf{a}_{i}\right\rangle_{i \in \mathbb{R}}$ such that $\left|S_{\varphi}(A)\right| \geq|A|^{d-\epsilon}$ for arbitrarily large finite $A \subseteq\left\langle\mathbf{a}_{i}\right\rangle_{i \in \mathbb{R}}$.

Claim $\exists B \in S_{\varphi}\left(\left\langle\mathbf{a}_{i}\right\rangle_{i \in \mathbb{R}}\right)$ with $\lg (\mathbb{G}(B))=d+1$.

First, we argue that there is $B \in S_{\varphi}\left(\left\langle\mathbf{a}_{i}\right\rangle_{i \in \mathbb{R}}\right)$ with $\lg (\mathbb{G}(B)) \geq d+1$. Suppose, to the contrary, that $\forall B \in S_{\varphi}\left(\left\langle\mathbf{a}_{i}\right\rangle_{i \in \mathbb{R}}\right)$, we have $\lg (\mathbb{G}(B)) \leq d$. Let $A \subseteq\left\langle\mathbf{a}_{i}\right\rangle_{i \in \mathbb{R}}$ be finite, and consider an arbitrary $B \in S_{\varphi}\left(\left\langle\mathbf{a}_{i}\right\rangle_{i \in \mathbb{R}}\right)$. There are $2^{d+1}-1$ possibilities for $\mathbb{G}(B)$. For any choice of $\mathbb{G}(B)$, by Theorem 2.7 ,

$$
\left|\left\{C \cap A: C \in S_{\varphi}\left(\left\langle\mathbf{a}_{i}\right\rangle_{i \in \mathbb{R}}\right), \mathbb{G}(C)=\mathbb{G}(B)\right\}\right| \leq\left(\begin{array}{c}
|A| \\
\leq d-1
\end{array}\right) .
$$

These two facts imply that $\left|S_{\varphi}(A)\right| \leq\left(2^{d+1}-1\right) \cdot\left(\begin{array}{c}|A| \\ \leq d-1\end{array}\right)=O\left(|A|^{d-1}\right)$. Because this holds for any finite $A \subseteq\left\langle\mathbf{a}_{i}\right\rangle_{i \in \mathbb{R}}$, the hypothesis on $\left\langle\mathbf{a}_{i}\right\rangle_{i \in \mathbb{R}}$ is violated.

We now assume that $B \in S_{\varphi}\left(\left\langle\mathbf{a}_{i}\right\rangle_{i \in \mathbb{R}}\right)$ and that $\lg (\mathbb{G}(B)) \geq d+1$. Without loss of generality, $\varphi$ is NIP, because otherwise we have $\operatorname{VCm}(\varphi)=\operatorname{VCd}_{\text {ind }}(\varphi)=\infty$. Under these assumptions, $\mathbb{G}(B)=n \geq d+1$ for some $n \in \omega$. Inducting on $n$, it follows by compactness and saturation of the monster that there is some $B^{\prime} \in S_{\varphi}\left(\left\langle\mathbf{a}_{i}\right\rangle_{i \in \mathbb{R}}\right)$, with $\lg \left(\mathbb{G}\left(B^{\prime}\right)\right)=d+1$. This proves the claim.

Now take $B$ as in the claim. It follows from the transfer lemma that on any finite $A \subseteq\left\langle\mathbf{a}_{i}\right\rangle_{i \in \mathbb{R}}$,

$$
\left\{B^{\prime} \cap A: B^{\prime} \subseteq\left\langle\mathbf{a}_{i}\right\rangle_{i \in \mathbb{R}}, \mathbb{G}\left(B^{\prime}\right)=\mathbb{G}(B)\right\} \in \operatorname{Tr}(\varphi, A) .
$$

This implies, by Theorem 2.7, that $\varphi$ traces arbitrarily large $d$-maximum set systems, and, by Lemma 3.4, $\operatorname{Tr}_{\aleph_{0}}(\varphi)$ contains a $d$-maximum set system. Thus $\operatorname{VCm}(\varphi) \geq d$.

To show the other direction, suppose that $\operatorname{VCm}(\varphi) \geq d$. By compactness, saturation, and Ramsey's theorem (or, alternatively, by the Erdös-Rado theorem), there is an infinite indiscernible sequence $A=\left\langle\mathbf{a}_{i}\right\rangle_{i \in \omega}$ on which $\varphi$ traces a $d$-maximum set system. It follows from the definition of $d$-maximum that $S_{\varphi}(A)$ witnesses that $\operatorname{VCd}_{\text {ind }}(\varphi) \geq d$. 
It should be noted that Guingona and Hill prove that $\operatorname{VCd}_{\text {ind }}(\varphi)$ is equal to several other invariants, among which $\operatorname{VCm}(\varphi)$ may obviously be included.

An immediate corollary is the following.

Corollary 3.8 For any formula $\varphi(\mathbf{x}, \mathbf{y}), \operatorname{VCd}(\varphi)=\operatorname{VCd}_{\text {ind }}(\varphi)$ if and only if $\varphi$ traces an infinite $d$-maximum set system, where $d=\operatorname{VCd}(\varphi)$.

This condition is easier to use in practice than a direct appeal to a nonconstructive combinatorial principle such as the Ramsey or Erdös-Rado theorem. We give an algebraic example in the theory of real closed fields (RCF). Though we make efforts to be precise in the following statements, we are just considering a definable family that results from a polynomial inequality where the coefficients form the parameter set.

Without loss of generality we work in $\mathbb{R}$. Let $\mathbf{y}$ be a finite sequence of variables. For a given $m \in \omega$, let $Y_{m}$ denote the set of monomials which occur in the general polynomial of degree $m$ with variables $\mathbf{y}$. Consider a family of polynomials of the form

$$
p\left(c_{1}, \ldots, c_{d}, \mathbf{y}\right)=u_{0}(\mathbf{y})+c_{1} u_{1}(\mathbf{y})+\cdots+c_{d} u_{d}(\mathbf{y}),
$$

where for each $i=0, \ldots, d, u_{i}(\mathbf{y}) \in Y_{m}$ and for each $i=1, \ldots, d, c_{i} \in \mathbb{R}$. Define $\mathcal{C}=\left\{\operatorname{pos}(p(\mathbf{c}, \mathbf{y})): \mathbf{c} \in \mathbb{R}^{d}\right\}$, where $\operatorname{pos}(p(\mathbf{y}))=\left\{\mathbf{a} \in \mathbb{R}^{|\mathbf{y}|}: p(\mathbf{a}) \geq 0\right\}$.

Such a $\mathcal{C}$ can clearly be traced by some $\varphi(\mathbf{x}, \mathbf{y})=p(\mathbf{x}, \mathbf{y}) \in L_{\text {ring }}$, in $\mathbb{R} \models$ RCF. It is known (see Floyd [4, Section 8.1]) that for such a $\varphi$, we have $\operatorname{VCm}(\varphi) \geq d$. As it is well known that $\operatorname{VCd}(\varphi)=d$, we have $\operatorname{VCd}_{\text {ind }}(\varphi)=\operatorname{VCd}(\varphi)$ for polynomial inequalities $\varphi$.

Floyd's result is based on an application of Dudley's theorem (see [3, Theorem 4.2.1]), which can apply to somewhat more general situations (see Ben-David and Litman [2]).

Many familiar geometric families, such as circles, ellipses, positive half-spaces, hyperbolas, and so on, therefore have $\mathrm{VC}_{\text {ind }}$-density equal to VC-density. The above approach notably does not apply to geometric families which are not polynomially definable (in the above sense) such as axis-parallel rectangles, or convex $d$-gons.

3.2 Stability Here we show how to characterize the stability of $\varphi$ using the maximum systems in $\operatorname{Tr}(\varphi, A)$. (For a review of the relevant notions from stability theory, see Hodges [6].)

Recall that the ladder dimension of a formula $\varphi(\mathbf{x}, \mathbf{y})$ is defined by writing $\operatorname{LD}(\varphi) \geq n$ if and only if there are $\mathbf{a}_{0}, \ldots, \mathbf{a}_{n-1}$ in $\mathbf{M}^{|\mathbf{x}|}$ and $\mathbf{b}_{0}, \ldots, \mathbf{b}_{n-1}$ in $\mathbf{M}^{|\mathbf{y}|}$ such that $\mathfrak{M} \models \varphi\left(\mathbf{a}_{i}, \mathbf{b}_{j}\right) \Longleftrightarrow i<j$. Finite ladder dimension is equivalent to stability for formulas. The VC-dimension can be thought of as a generalization of ladder dimension, and in general $\operatorname{coVC}(\varphi) \leq \operatorname{LD}(\varphi)$, where $\operatorname{coVC}(\varphi)$ denotes the VC-dimension of $S_{\varphi}\left(\mathrm{M}^{|\mathbf{y}|}\right)$ conceived as a set family.

For a set $X$ and $\mathscr{C} \subseteq \mathcal{P}(X)$, define a graph $\mathscr{E}_{\mathcal{C}}=(V, E)$ where $V=\mathcal{C}$ and where $E\left(C_{1}, C_{2}\right)$ holds if and only if $\left|C_{1} \Delta C_{2}\right|=1$. For $C_{1}, C_{2} \in \mathcal{C}$, define $\operatorname{dist}_{h}\left(C_{1}, C_{2}\right)$ to be the Hamming distance $\left|C_{1} \Delta C_{2}\right|$, and $\operatorname{dist}_{G_{e}}\left(C_{1}, C_{2}\right)$ to be the graph distance in $\mathbb{S}_{e}$, with $\operatorname{dist}_{\mathfrak{F}_{e}}\left(C_{1}, C_{2}\right)=\infty$ if $C_{1}$ and $C_{2}$ belong to different components.

The following was proved by Kuzmin and Warmuth [10].

Lemma 3.9 ([10, Lemma 14]) Let $X$ be a finite set. Suppose that $\mathcal{C} \subseteq \mathcal{P}(X)$ is $d$-maximum and that $C_{1}, C_{2} \in \mathcal{C}$. Then 


$$
\operatorname{dist}_{h}\left(C_{1}, C_{2}\right)=\operatorname{dist}_{G_{e}}\left(C_{1}, C_{2}\right) .
$$

In particular, se is connected.

The equivalence of $\operatorname{dist}_{h}$ and dist $_{G_{e}}$ is clearly still true when $X$ is infinite, though the graph $5^{r} e$ will not be connected in general. In fact, in many natural maximum set systems (e.g., open intervals on a densely ordered set), (5e is totally disconnected.

Theorem 3.10 For $\varphi(\mathbf{x}, \mathbf{y})$ a formula, $\varphi$ is stable with $\operatorname{LD}(\varphi) \leq n$ if and only if for every $A \subseteq \mathrm{M}^{|y|}$ and every $\varphi \in \operatorname{Tr}(\varphi, A)$ which is $d$-maximum for some $d \in \omega$, for any $C_{1}, C_{2} \in \mathcal{C},\left|C_{1} \backslash C_{2}\right| \leq n$.

Proof First, suppose that $\varphi(\mathbf{x}, \mathbf{y})$ is a stable formula with $\operatorname{LD}(\varphi) \leq n$, and $A \subseteq \mathrm{M}^{|y|}$.

Let $C_{1}, C_{2} \in \mathcal{C}$, where $\ell \in \operatorname{Tr}(\varphi, A)$ is $d$-maximum for some $d \in \omega$. We will show that $\left|C_{1} \backslash C_{2}\right| \leq n$. Note that it suffices to consider the case in which $A$ is finite. Thus we may assume, by Lemma 3.9, that $G_{e} e$ is connected.

Let $\left\{a_{1}, \ldots, a_{k}\right\} \subseteq C_{1} \backslash C_{2}$ be a set of distinct elements. By Lemma 3.9, after possibly reordering, there are $B_{k}, B_{k-1}, \ldots, B_{1}$ in $\ell$, on the path from $C_{1}$ to $C_{2}$ in (5e, such that $a_{i} \in B_{j}$ iff $i<j$. Thus $k \leq n$, and consequently $\left|C_{1} \backslash C_{2}\right| \leq n$.

Conversely, suppose that $\operatorname{LD}(\varphi)>n$. Let $B=\left\{\mathbf{b}_{0}, \ldots, \mathbf{b}_{n}\right\} \subseteq \mathrm{M}^{|\mathbf{x}|}$, and define $A=\left\{\mathbf{a}_{0}, \ldots, \mathbf{a}_{n}\right\} \subseteq \mathbf{M}^{|\mathbf{y}|}$ such that $\mathfrak{M} \models \varphi\left(\mathbf{b}_{i}, \mathbf{a}_{j}\right) \Longleftrightarrow i<j$. Put $\mathcal{C}=\left.S_{\varphi}(A)\right|_{B}$. Then by Theorem 2.7, $\mathcal{C}$ is 1-maximum, and $|A \backslash \emptyset|=|A|=n+1$.

The above theorem shows that much of the nature of Shelah's famous characterization of stable formulas by indiscernibles (see [15]) is already visible at the level of maximum traces. Unspooling the theorem reveals a structural characterization of stable maximum set systems, as we now show.

If $X$ is a set, and $\mathcal{C} \subseteq \mathcal{P}(X)$, define the ladder dimension of $\mathcal{C}$ to be the maximal $n \in \omega$ such that there are $x_{1}, \ldots, x_{n} \in X$ and $B_{1}, \ldots, B_{n} \in \mathcal{C}$ with $x_{i} \in B_{j}$ if and only if $i<j$. Say that $\zeta$ is stable just in case it has finite ladder dimension.

If $A \subseteq X$, define $\mathcal{C} \Delta=\{C \Delta A: C \in \mathcal{C}\}$.

Lemma 3.11 If $\mathcal{C} \subseteq \mathcal{P}(X)$ has $\operatorname{LD}(\mathcal{C})=n$, then for any $A \subseteq X$, we have $\mathrm{LD}(\mathcal{C} \Delta A) \leq 2 n$, and this bound is tight.

Proof Suppose that $\operatorname{LD}(\mathcal{C} \Delta A)=2 n$ for an integer $n$. Then there exist $x_{1}, \ldots, x_{2 n}$ in $X$ and $C_{1}, \ldots, C_{2 n} \in \mathcal{C}$ such that for all $i, j \leq 2 n, x_{i} \in C_{j} \Delta A$ if and only if $i<j$. Consider the case in which there are indices $i_{1}<\cdots<i_{n}$ such that for each $k=1, \ldots, n, x_{i_{k}} \notin A$. Then these elements, together with an appropriate choice of $C_{j_{1}}, \ldots, C_{j_{n}}$, witness that $\operatorname{LD}(\mathcal{C}) \geq n$. Now suppose that the opposite holds, namely, that there are indices $i_{1}<\cdots<i_{n+1}$ such that for each $k=1, \ldots, n+1$, $x_{i_{k}} \in A$. Then $i_{k}<j$ and $x_{i_{k}} \notin C_{j}$ are equivalent, since both are equivalent to $x_{i_{k}} \in C_{j} \Delta A$. Reindexing by $C_{j}^{\prime}=C_{2 n-j}$ and taking an appropriate $j_{1}<\cdots<j_{n}$, we have that $x_{i_{k}} \in C_{j_{l}}^{\prime}$ if and only if $k<l$. Thus $\operatorname{LD}(\mathcal{C}) \geq n$. A similar argument shows that $\operatorname{LD}(\mathcal{C}) \geq n$ in the case in which $\operatorname{LD}(\mathcal{C} \Delta A)=2 n+1$ is odd. This establishes the bound.

To see that the bound is tight, fix $n \in \omega$. Let $X$ be the integers between $-n$ and $n$, inclusive, but not including zero. Let $\mathcal{C}=\{[0, i] \cap X: 0<i \leq n\} \cup\{[-i, 0] \cap X$ : $0<i \leq n\}$. Clearly $\operatorname{LD}(\mathcal{C})=n$. But $\operatorname{LD}(\mathcal{C} \Delta[-n,-1])=2 n$. 
Note that the example in the above proof is 1-maximum.

Corollary 3.12 Let $\mathcal{C} \subseteq \mathcal{P}(X)$ be d-maximum of ladder dimension $n$.

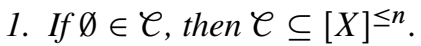

2. $\varphi \Delta B \subseteq[X] \leq 2 n$ for any $B \in \mathcal{C}$.

3. $\varphi \subseteq[X]^{\leq 2 n} \Delta B$ for any $B \in \mathcal{C}$.

Proof The claim in (1) is clear from Theorem 3.10. For the claim in (2) note that $\emptyset \in \mathcal{C} \Delta B$ (because $B \in \mathcal{C}$ ), and the ladder dimension of $\mathcal{C} \Delta B$ is at most $2 n$ by Lemma 3.11. Therefore $\mathcal{C} \Delta B \subseteq[X]^{\leq 2 n}$ by (1). Claim (3) follows after applying $\Delta B$ to both sides of the containment in (2).

The $2 n$ bound in Corollary 3.12(2) is tight, as the following example shows. Let $X=\{1, \ldots, 2 n\}$, and let $\ell=[X]^{\leq n}$. Clearly $\operatorname{LD}(\mathcal{C})=n$. Now putting $B=\{1, \ldots, n\}$ gives $\mathcal{C} \Delta B$ an element of cardinality $2 n$.

On the other hand, it seems possible that for some $B \subseteq X$, it holds that $\ell \Delta B \subseteq[X]^{\leq n}$, where the hypotheses are as in Corollary 3.12. However, since the hypotheses admit all finite maximum classes, this conjecture may be too optimistic. Such a result would clearly be the best possible.

It is also evident from the above that the stable maximum set systems are exactly those maximum set systems $\ell \subseteq \mathcal{P}(X)$ which can be realized as $\mathcal{C} \subseteq[X]^{m} \Delta B$ for some $m \in \omega$ and $B \subseteq X$ (because the latter systems are clearly stable).

It would be useful to know whether every $\mathcal{C}$ of ladder dimension $n$ embeds into a $O(n)$-maximum set system $\ell^{\prime}$ (see [2] for relevant embedding notions). It is conjectured (see [4]) that the vast majority of set systems are not embeddable in maximum systems of the same VC-dimension, prompting the question of whether stable set systems, which are well behaved in so many respects, are also unusual in this way. Very little is known about model-theoretic criteria for when a definable family embeds in a maximum family, other than the easy observation that this is frequently possible in dimension 1.

Many nice properties of maximum set systems, in particular the existence of compression schemes (see [10] for definitions), are inherited under the relation of embedded substructure. Compression schemes emphasize the amount of information needed to represent a $\varphi$-type rather than the definability of the representation, and as a consequence they can be used to bound not only the VC-density of a set system, but also the size of the multiplicative constant in the definition of VC-density.

\section{Conclusion}

In model theory, much of the combinatorial content of theories comes from considering formulas restricted to indiscernible sequences. The existence of sequences of indiscernibles is guaranteed by Ramsey's theorem (and compactness), though it is rarely required to exhibit a concrete sequence of indiscernibles.

When dealing with a certain formula $\varphi(\mathbf{x}, \mathbf{y})$ on a sequence $A=\left\langle\mathbf{a}_{i}\right\rangle_{i \in I}$, it is a weaker condition to assume that $\varphi$ is maximum on $A$ than to assume that $A$ is indiscernible. However, as we have seen, if $\varphi$ is maximum on $A$, that provides "enough" indiscernibility for some combinatorial notions to manifest. Namely, $d p$-rank, NIP, and stability can all be understood in terms of the maximum property. Avoiding the use of indiscernibles has the potential to make these concepts, particularly $d p$-rank, much more accessible to non-model theorists. 
Unlike indiscernible sequences, maximum domains are frequently easily constructible. In the semilinear case, it follows from the work of Floyd and Dudley that a basic semilinear family will be maximum on a set of points which is in "general position," for which it is sufficient to take a randomly selected set of points.

Moreover, the similarity of maximum families and formulas on indiscernible sequences provides a point of contact between work done in computational learning theory and model theory, where, especially recently, researchers are pursuing compatible combinatorial goals, but without a common framework.

\section{References}

[1] Aschenbrenner, M., A. Dolich, D. Haskell, H. D. Macpherson, and S. Starchenko, "Vapnik-Chervonenkis density in some theories without the independence property, II," Notre Dame Journal of Formal Logic, vol. 54 (2013), pp. 311-63. MR 3091661. DOI 10.1215/00294527-2143862. 583, 586

[2] Ben-David, S., and A. Litman, "Combinatorial variability of Vapnik-Chervonenkis classes with applications to sample compression schemes," Discrete Applied Mathematics, vol. 86 (1998), pp. 3-25. Zbl 0905.68048. MR 1634863. DOI 10.1016/S0166-218X(98)00000-6. 589, 591

[3] Dudley, R. M., Uniform Central Limit Theorems, vol. 63 of Cambridge Studies in Advanced Mathematics, Cambridge University Press, Cambridge, 1999. MR 1720712. DOI 10.1017/CBO9780511665622. 589

[4] Floyd, S., "Space-bounded learning and the Vapnik-Chervonenkis dimension," pp. 349-64 in Proceedings of the Second Annual Workshop on Computational Learning Theory (Santa Cruz, Calif., 1989), Morgan Kaufmann, San Mateo, Calif., 1989. MR 1076240. 584, 585, 589, 591

[5] Guingona, V., and C. D. Hill, "On VC-density over indiscernible sequences," preprint, arXiv: $1108.2554 \mathrm{v} 4$ [math.LO]. 583

[6] Hodges, W., A Shorter Model Theory, Cambridge University Press, Cambridge, 1997. MR 1462612. 589

[7] Kaplan, I., A. Onshuus, and A. Usvyatsov, "Additivity of the dp-rank," Transactions of the American Mathematical Society, vol. 365 (2013), pp. 5783-804. MR 3091265.583

[8] Johnson, H. R., "Dp-rank and forbidden configurations," Notre Dame Journal of Formal Logic, vol. 54 (2013), pp. 1-15. Zbl 1276.03035. MR 3007957. DOI 10.1215/00294527-1731335. 585

[9] Johnson, H. R., and M. C. Laskowski, "Compression schemes, stable definable families, and o-minimal structures," Discrete and Computational Geometry, vol. 43 (2010), pp. 914-26. Zbl 1201.03022. MR 2610477. DOI 10.1007/s00454-009-9201-3. 586

[10] Kuzmin, D., and M. K. Warmuth, "Unlabeled compression schemes for maximum classes," Journal of Machine Learning Research, vol. 8 (2007), pp. 2047-81. Zbl 1222.68239. MR 2353827. DOI 10.1007/11503415_40. 584, 589, 591

[11] Laskowski, M. C., "Vapnik-Chervonenkis classes of definable sets," Journal of the London Mathematical Society (2), vol. 45 (1992), pp. 377-84. MR 1171563. DOI 10.1112/j1ms/s2-45.2.377. 584

[12] Rubinstein, B. I. P., and J. H. Rubinstein, "A geometric approach to sample compression," Journal of Machine Learning Research, vol. 13 (2012), pp. 1221-61. MR 2930638. 584

[13] Sauer, N., "On the density of families of sets," Journal of Combinatorial Theory Series A, vol. 13 (1972), pp. 145-47. Zbl 0248.05005. MR 0307902. 584

[14] Shelah, S., "A combinatorial problem: Stability and order for models and theories in infinitary languages," Pacific Journal of Mathematics, vol. 41 (1972), pp. 247-61. 
Zbl 0239.02024. MR 0307903. 584

[15] S. Shelah Classification Theory and the Number of Nonisomorphic Models, 2nd edition, vol. 92 of Studies in Logic and the Foundations of Mathematics, North-Holland, Amsterdam, 1990. MR 1083551. 590

[16] Vapnik, V., and A. Chervonenkis, "On the uniform convergence of relative frequencies of events to their probabilities," Theory of Probability and its Applications, vol. 16 (1971), pp. 264-80. Zbl 0247.60005. 584

[17] Welzl, E., “Complete range spaces,” unpublished notes, 1987. 584

\section{Acknowledgments}

Many thanks to the referee and to Vince Guingona for comments on an early version of this paper. This research was partially supported by Professional Staff Congress-City University of New York grant 64679-00 42.

Department of Mathematics and Computer Science John Jay College, City University of New York

New York, New York, USA

hujohnson@jjay.cuny.edu

http://jjcweb.jjay.cuny.edu/hjohnson/ 